\title{
Diurnal and Sub-diurnal Terms of Nutation
}

\author{
Aleksander Brzeziński \\ Space Research Centre, Polish Academy of Sciences, Bartycka 18A, \\ 00-716 Warsaw, Poland, e-mail: alek@cbk.waw.pl
}

\begin{abstract}
This paper discusses the short-period terms of nutation which are included in the recent theories of nutation for a rigid Earth. We argue that these terms should be expressed as terrestrial perturbations, that is as polar motion, and describe how such a transformation can be accomplished in practice. Based on the available rigid-Earth amplitudes, we estimate this lunisolar effect in polar motion for an elastic Earth with liquid core, and compare it to the oceanic and atmospheric perturbations at similar frequencies.
\end{abstract}

\section{Introduction}

Recent developments of the rigid-Earth nutation theory, such as SMART97 (Bretagnon et al., 1998), REN2000 (Souchay et al., 1999), RDAN97 (Roosbeek, 1999), contain short-period terms caused by the interaction of the lunisolar tidal potential with those geopotential components which express departures from rotational symmetry. These are quasi-diurnal nutations corresponding to the $C_{3,1}$, $S_{3,1}, C_{4,1}, S_{4,1}$ components of geopotential, quasi-semidiurnal nutations corresponding to the $C_{2,2}, S_{2,2}, C_{3,2}, S_{3,2}$ components, and quasi-terdiurnal nutations corresponding to the $C_{3,3}, S_{3,3}$ components. Most of these nutations are prograde (counterclockwise), the largest retrograde amplitudes being of the order of $0.5 \mu$ as (microarcsecond). Therefore, when expressed in the terrestrial frame they become the low frequency (prograde and retrograde), diurnal prograde and semidiurnal prograde variations, respectively, in polar motion. This part of the nutation spectrum has received some attention recently (e.g. Bretagnon, 1998; Bizouard et al., 2000), nevertheless it is not included in the nonrigid-Earth nutation models.

The aim of this paper is to describe short-period nutation and to study its importance for the astrometric determination of the Earth orientation parameters and for their geophysical interpretation. We start from the kinematical considerations which describe how the diurnal and sub-diurnal terms of nutation can be expressed as polar motion. Next, we estimate how their amplitudes are modified by the nonrigid-Earth response. Finally, we compare this part of the lunisolar effect on the equatorial component of Earth rotation with the oceanic and atmospheric perturbations at similar frequencies. 


\section{Kinematical relationships}

Current observational practice is to express perturbations in Earth rotation by the following set of the Earth orientation parameters $\left\{x_{p}, y_{p}, \delta \psi, \delta \varepsilon, \mathrm{UT} 1-\mathrm{UTC}\right\}$, where $x_{p},-y_{p}$ denote the terrestrial coordinates of the Celestial Ephemeris Pole (CEP), $\delta \psi, \delta \varepsilon$ are the spatial offsets in longitude and obliquity of the CEP with respect to its position predicted by the precession/nutation model, and UT1-UTC denotes the variation of Universal Time (McCarthy, 1996). We will consider polar motion as a terrestrial motion of the CEP and denote it $p=x_{p}-i y_{p}$, and nutation as a residual (that is with respect to the conventional a priori model) celestial motion $P=X+i Y=\sin \varepsilon_{0} \delta \psi+i \delta \varepsilon$, where $i=\sqrt{-1}$ denotes the imaginary unit and $\varepsilon_{o}$ is the mean obliquity at the reference epoch. For more details concerning this parameterization of Earth rotation see Brzeziński and Capitaine (1993).

\subsection{Nutation versus polar motion}

There is a full degeneracy between polar motion and nutation parameters: residual nutation $P$ (or its part) can be equivalently expressed as polar motion $\Delta p$ using the following first-order relationship (Brzeziński and Capitaine, 1993)

$$
\Delta p=-P e^{-i \theta},
$$

which should be understood in a sense that new polar motion coordinates are $p+\Delta p$. Here $\theta=\Omega t+\theta_{o}(t)$ is the rotation angle around the CEP axis, where $\Omega$ is an average angular speed of diurnal sidereal rotation and $\theta_{0}$ is a quasi-constant phase referred to $t=0$.

A single quasi-harmonic term of nutation is usually expressed as

$$
\begin{aligned}
\Delta \psi & =\psi_{s} \sin \gamma(t)+\psi_{c} \cos \gamma(t), \\
\Delta \varepsilon & =\varepsilon_{s} \sin \gamma(t)+\varepsilon_{c} \cos \gamma(t),
\end{aligned}
$$

(e.g. Souchay et al., 1999), where the nutation argument

$$
\gamma(t)=k_{1} l_{m}+k_{2} l_{s}+k_{3} F+k_{4} D+k_{5} \hat{\Omega}+k_{6} \phi
$$

is integer combination of the fundamental arguments: $l_{m}$ - the mean anomaly of the Moon, $l_{s}$ - the mean anomaly of the Sun, $F$ - the difference between the mean longitude of the Moon and the mean longitude of its node, $D$ - the difference between the mean longitudes of the Moon and of the Sun, $\hat{\Omega}-$ the mean longitude of the node of the moon, and of the Greenwich mean sidereal time (GMST) $\phi$. This argument takes the form $\gamma(t)=\lambda t+\gamma_{o}(t)$ where $\lambda$ is the angular frequency of nutation and $\gamma_{0}(t)$ a quasi-constant phase. For $k_{6}=0$ we have classical long periodic nutation components, while for $k_{6}=1,2,3$ - diurnal, semidiurnal and terdiurnal components of nutation, respectively.

The corresponding perturbation of the CEP is an elliptical celestial motion which can be expressed as a sum of two quasi-uniform circular oscillations with the same period but in the opposite directions

$$
P=\sin \varepsilon_{0} \Delta \psi+i \Delta \varepsilon=a_{+} e^{i \varphi_{+}} e^{i \gamma(t)}+a_{-} e^{i \varphi_{-}} e^{-i \gamma(t)},
$$


where $a>0$ denotes the amplitude, $\varphi$ is the phase with respect to the argument $\gamma$. If $\lambda>0$, which is the case for the short-period nutation, the term labelled by the subscript " + " describes the prograde (counterclockwise) motion, while that with the subscript "-" the retrograde (clockwise) motion. From the comparison of eq. (5) to equations (2), (3) we derive

$$
\begin{array}{ll}
a_{+} \cos \varphi_{+}=0.5\left(\psi_{c} \sin \varepsilon_{o}+\varepsilon_{s}\right), & a_{+} \sin \varphi_{+}=0.5\left(-\psi_{s} \sin \varepsilon_{o}+\varepsilon_{c}\right), \\
a_{-} \cos \varphi_{-}=0.5\left(\psi_{c} \sin \varepsilon_{o}-\varepsilon_{s}\right), & a_{-} \sin \varphi_{-}=0.5\left(\psi_{s} \sin \varepsilon_{o}+\varepsilon_{c}\right) .
\end{array}
$$

For the diurnal and sub-diurnal nutation terms the retrograde amplitudes are much smaller than the prograde ones, the largest being about $0.6 \mu \mathrm{as}$, therefore we will neglect them retaining in eq. (5) only the prograde term and dropping the subscript " + " for simplicity. After neglecting small differences between $\theta$ and $\phi$, eq. (1) yields the following terrestrial representation of the nutation

$$
\Delta p=\Delta x_{p}-i \Delta y_{p}=a e^{i(\varphi+\pi)} e^{i \gamma^{\prime}(t)}
$$

with

$$
\gamma^{\prime}(t)=\gamma(t)-\phi=k_{1} l_{m}+k_{2} l_{s}+k_{3} F+k_{4} D+k_{5} \hat{\Omega}+\left(k_{6}-1\right) \phi .
$$

Hence, in the conventional representation of nutation, the transformation from the celestial to the terrestrial representation is very simple: it consists of decreasing the coefficient of GMST in $\gamma$ by 1 and reversing the phase $\varphi$.

\subsection{CEP versus instantaneous pole of rotation}

Let $\vec{\omega}=\Omega\left(m_{1}, m_{2}, 1+m_{3}\right)^{T}$ be the Earth's instantaneous rotation vector expressed in the terrestrial reference system (TRS), where $m_{l}$ are small dimensionless quantities introduced by Munk and MacDonald (1960). Any perturbation of the corresponding pole, the instantaneous pole of rotation, $m=m_{1}+i m_{2}$ can be related to the reported quantity, polar motion $p$ of the CEP, using the following first-order relationship derived by Brzeziński (1992)

$$
m=p-i \frac{\dot{p}}{\Omega}
$$

or in the frequency domain representation

$$
m_{\sigma} e^{i \sigma t}=\frac{\sigma+\Omega}{\Omega} p_{\sigma} e^{i \sigma t},
$$

where $\sigma$ denotes the Earth-referred angular frequency and $m_{\sigma}, p_{\sigma}$ are amplitudes, complex in general. See also Brzeziński and Capitaine (1993) for discussion.

For low-frequency polar motion the differences between the amplitudes $m_{\sigma}$ and $p_{\sigma}$ are small, but for the prograde diurnal and semidiurnal variations the ratio $p_{\sigma} / m_{\sigma}=(1+\sigma / \Omega)^{-1}$ equalling $1 / 2,1 / 3$, respectively. 


\section{Lunisolar excitation of polar motion}

Let us denote by $L=L_{1}+i L_{2}$ the equatorial component of the external gravitational torque on the Earth, expressed in the TRS, which is responsible for polar motion. Let us further assume that it takes a harmonic form

$$
L=L_{\sigma} e^{i \sigma t},
$$

where in the present case the frequency $\sigma$ is in the vicinity of $0, \Omega$ or $2 \Omega$.

We will consider a very simple model of the Earth consisting of the rotationally symmetrical elastic mantle and the liquid core, with no coupling between these two. Note, however, that this model is fully consistent with that applied to estimate the corresponding long-period and diurnal prograde atmospheric and oceanic influence on polar motion: (See e.g. Brzeziński and Petrov, 2000; Chao et al., 1991; 1996; Gross et al., 1998). The elastic yielding of the mantle is a sum of two different effects (Munk and MacDonald, 1960):

- rotational deformation proportional to the polar motion amplitude $m$ and appearing independently of the physical nature of perturbation, and

- tidal deformation associated with external gravitational forcing and proportional to the torque $L$.

After taking into account these effects, the corresponding polar motion solution is obtained in the following form

$$
m=m_{\sigma} e^{i \sigma t},
$$

with the (complex) amplitude expressed by

$$
m_{\sigma}=-\frac{i}{\sigma-\sigma_{e}} \frac{1}{A_{m} \Omega} \frac{1-\frac{k}{k_{s}} \frac{\sigma+\Omega}{\Omega}}{1+\frac{k}{k_{s}} e_{m}} L_{\sigma},
$$

where $k$ denotes the tidal Love number, $k_{s}=0.94$ is the secular Love number, $e_{m}=\left(C_{m}-A_{m}\right) / A_{m}$ stands for the dynamical ellipticity of the mantle, $A_{m}, C_{m}$ are the principal equatorial and axial moments of inertia of the unperturbed mantle, and

$$
\sigma_{e}=e_{m} \frac{1-\frac{k}{k_{s}}}{1+\frac{k}{k_{s}} e_{m}} \Omega
$$

is the Chandler frequency of the mantle. This is eq. (3-146) of Moritz and Mueller (1987) in which the principal moments $A, C$ of the entire Earth have been replaced by those confined to the mantle alone.

We can compare this solution to the corresponding solution for the rigid Earth, that is the case $k=0, A_{m}=A, C_{m}=C$, by the ratio

$$
\frac{m_{\sigma}}{m_{\sigma}^{r}}=q(\sigma) \frac{L_{\sigma}}{L_{\sigma}^{r}}
$$

in which the nonrigid Earth response function $q(\sigma)$ is given by

$$
q(\sigma)=\frac{\sigma-\sigma_{r}}{\sigma-\sigma_{e}} \frac{A}{A_{m}} \frac{1-\frac{k}{k_{s}} \frac{\sigma+\Omega}{\Omega}}{1+\frac{k}{k_{s}} e_{m}},
$$


where $\sigma_{r}$ is the Euler frequency of resonance for the rigid Earth, $L_{\sigma}^{r}$ is the lunisolar torque on the entire Earth, and $L_{\sigma}$ is that on the mantle.

Let us first consider separately various contributions to $q(\sigma)$ :

1. Elastic rotational deformations proportional to $m$ :

- Change of the resonant frequency from $\sigma_{r}=\Omega / 304$ to $\sigma_{e}=\Omega / 430$. The corresponding factor $\left(\sigma-\sigma_{r}\right) /\left(\sigma-\sigma_{e}\right)$ differs significantly from unity only at low frequencies.

- Factor $\left[1+\left(k / k_{s}\right) e_{m}\right]^{-1} \approx 0.999$ which is not important.

2. Elastic tidal deformations (proportional to $L$ ):

- Factor $1-\left(k / k_{s}\right)(\sigma+\Omega) / \Omega$ which equals about $0.7,0.4,0.1$ for $\sigma=0, \Omega, 2 \Omega$, respectively. Note that for the long periodic nutations $(\sigma \approx-\Omega)$ this factor is close to 1 .

3. Decoupling of the liquid core:

- Factor $A / A_{m}=1.129$.

Table 1. Nonrigid Earth response functions: W81 - (Wahr, 1981); DD1, DD2 - (Dehant and Defraigne, 1997), eq. (4), eq. (5), respectively; M91 - (Mathews et al., 1991). All the functions have been modified by adjusting the Chandler frequency to the observed value of 1 cycle in $\mathbf{4 3 3}$ days. Periods are in solar days and amplitudes in microarcseconds.

\begin{tabular}{|c|c|c|c|c|c|c|c|}
\hline \multirow{2}{*}{$\begin{array}{c}\text { Celestial } \\
\text { period }\end{array}$} & \multirow{2}{*}{$\begin{array}{c}\text { Terrestrial } \\
\text { period }\end{array}$} & \multirow{2}{*}{$\begin{array}{l}\text { Rigid } \\
\text { ampl. }\end{array}$} & \multicolumn{5}{|c|}{ Nonrigid Earth response coefficients } \\
\hline & & & This work & W81 & DD1 & M91 & DD2 \\
\hline 1.07545 & -13.719 & 1.23 & 0.825 & 0.797 & 0.803 & 0.799 & 0.800 \\
\hline 1.03505 & -27.322 & 13.60 & 0.822 & 0.796 & 0.801 & 0.796 & 0.797 \\
\hline 1.00243 & -193.56 & 0.63 & 0.895 & 0.866 & 0.873 & 0.850 & 0.854 \\
\hline 0.99772 & -2190.4 & 1.22 & 1.070 & 1.033 & 1.041 & 0.981 & 0.991 \\
\hline 0.99758 & -3231.5 & 7.98 & 1.086 & 1.048 & 1.056 & 0.993 & 1.004 \\
\hline 0.99727 & $\infty$ & 0.74 & 1.125 & 1.086 & 1.094 & 1.023 & 1.035 \\
\hline 0.99696 & 3231.5 & 9.61 & 1.177 & 1.135 & 1.144 & 1.062 & 1.075 \\
\hline 0.99682 & 2190.4 & 1.62 & 1.208 & 1.164 & 1.174 & 1.083 & 1.100 \\
\hline 0.99455 & 365.24 & 0.89 & -1.025 & -0.962 & -0.975 & -0.590 & -0.653 \\
\hline 0.99425 & 328.17 & 0.53 & -0.264 & -0.237 & -0.243 & -0.019 & -0.056 \\
\hline 0.99216 & 193.56 & 2.83 & 0.516 & 0.506 & 0.508 & 0.566 & 0.557 \\
\hline 0.96215 & 27.322 & 15.27 & 0.755 & 0.734 & 0.738 & 0.744 & 0.743 \\
\hline 0.92969 & 13.719 & 1.15 & 0.754 & 0.734 & 0.738 & 0.742 & 0.742 \\
\hline 0.52743 & 1.11951 & 2.36 & 0.486 & 0.495 & 0.475 & 0.521 & 0.522 \\
\hline 0.49863 & 0.99727 & 14.31 & 0.449 & 0.461 & $\begin{array}{l}\text { olation } \\
0.436\end{array}$ & $\begin{array}{l}\text { plies .. } \\
0.490\end{array}$ & 0.492 \\
\hline 0.34502 & 0.52752 & 0.06 & 0.147 & 0.172 & 0.093 & 0.241 & 0.243 \\
\hline 0.33652 & 0.50790 & 0.05 & 0.122 & 0.147 & 0.063 & 0.220 & 0.223 \\
\hline
\end{tabular}

Our $q$ is compared in Table 1 to the response functions used in the recent nonrigid-Earth nutation theories, derived for more realistic multi-layer structural models of the Earth. These functions are well-constrained at the frequencies of the long-period nutations, which has been verified by comparison with the nutation amplitudes observed by VLBI, but this is not necessarily the case for the short-period nutations. In any case, our $q$ is consistent with other estimates to a few per cent. When expressed in terms of the corresponding nonrigid-Earth 
nutation amplitudes, the differences are below $0.5 \mu$ as which is a cut-off level adopted in Table 2.

Let us make the following remarks. First, when computing lunisolar effects on polar motion based on the rigid-Earth amplitudes $p_{\sigma}^{r}$ for the figure axis, which is the present case, the same response coefficient is applicable for the instantaneous rotation axis (Wahr, 1981; Moritz and Mueller, 1987)

$$
\frac{m_{\sigma}}{m_{\sigma}^{r}}=\frac{p_{\sigma}}{p_{\sigma}^{r}} .
$$

Secondly, the lunisolar torques estimates are based on the satellite-determined geopotential of the entire Earth, that is to a good accuracy our $L_{\sigma}^{r}$. When adopting the assumption that the core does not participate in rotational variations, the coefficient $L_{\sigma} / L_{\sigma}^{r}$ contains information about the large-scale features of the core-mantle boundary shape. Therefore, constraining the amplitudes of diurnal and sub-diurnal nutations from space geodesy observations, which is in fact empirically determining the ratio $L_{\sigma} / L_{\sigma}^{r}$, would provide important information about the internal constitution of the Earth.

\section{Results and comparison with oceanic and atmospheric effects}

Now, we will consider in detail diurnal and semidiurnal nutation components expressed as low frequency and prograde diurnal variations in polar motion, respectively. We will disregard terdiurnal nutation components because their amplitudes are below $0.1 \mu$ as for a rigid Earth, and according to Table 1 this number is further decreased about 8 times for an elastic Earth with liquid core.

\subsection{Prograde diurnal polar motion}

Prograde diurnal polar motion corresponding to semidiurnal nutation, is caused by the interaction of the lunisolar tidal potential with the $C_{n, 2}, S_{n, 2}(n \geq 2)$ terms of geopotential, where only the $n=2$ contributions are larger than $0.15 \mu$ as. This phenomenon was described and estimated for the first time by Chao et al. (1991) as the so-called "prograde diurnal libration" in polar motion. It is driven by the lunisolar torque taking the following form (see ibid.)

$$
L=-\frac{3 i G M(B-A)}{4 r^{3}} \sin 2 \Theta e^{-i\left(\Lambda-\Lambda_{o}\right)},
$$

where $G$ denotes gravitational constant, $M$ is the disturbing point mass, $r, \Theta, \Lambda$ are its geocentric radius, co-latitude and longitude, respectively, $B>A$ are the principal equatorial moments of the Earth's inertia, and $\Lambda_{o}$ denotes the longitude of the $A$-axis.

Our equations (14) and (11) with $\sigma=\Omega$ yield immediately the following solution

$$
p=-0.168 \frac{B-A}{A} \frac{G M}{r^{3} \Omega^{2}} \sin 2 \Theta e^{-i\left(\Lambda-\Lambda_{\circ}\right)},
$$

which describes prograde a nearly diurnal oscillation because $\Lambda \approx-\Omega t+$ const. Note, however, that Chao et al. $(1991 ; 1996)$ estimated the coefficient 0.36 instead of our 0.168 , which is also reflected in the individual amplitudes given in 
Table 2. This could be either due to the fact that their solution is for $m$ instead of the observed $p$ (see Sec. 2.2), or that they neglected the influence of the elastic tidal deformation on $q$ (see Sec. 3), both effects giving a factor of about 2 .

This prograde diurnal variation in polar motion is proportional to the equatorial dynamical flattening $(B-A) / A$ of the mantle, which can differ from that of the entire Earth by as much as $40 \%$ (Herring and Dong, 1994). If it was possible to estimate its amplitude from the observation of polar motion, we could constrain in this way the $C_{2,2}, S_{2,2}$ components of the core-mantle boundary.

Table 2. Largest long periodic and diurnal prograde variations in polar motion (amplitude> $0.5 \mu$ as) corresponding to diurnal and semidiurnal nutations from the REN2000 tables (Souchay et al., 1999). The last column shows estimates of Chao et al. $(1991,1996)$ (only of the amplitude because the phase is the same as in our estimate). Periods are in solar days and amplitudes in microarcseconds.

\begin{tabular}{|c|c|c|c|c|c|c|c|c|c|c|c|}
\hline \multicolumn{5}{|c|}{ Fundamental arguments } & \multirow[b]{2}{*}{$\phi$} & \multirow{2}{*}{$\begin{array}{l}\text { Tidal } \\
\text { code }\end{array}$} & \multirow{2}{*}{$\begin{array}{l}\text { Terrestr. } \\
\text { period }\end{array}$} & \multicolumn{2}{|c|}{ Amplitudes } & \multirow[b]{2}{*}{ Phase } & \multirow{2}{*}{$\begin{array}{l}\text { Chao } \\
\text { et al. }\end{array}$} \\
\hline$l_{m}$ & $l_{s}$ & $F$ & $D$ & $\hat{\Omega}$ & & & & rigid & elastic & & \\
\hline-1 & 0 & -1 & 0 & -1 & 0 & & -13.719 & 1.23 & 1.02 & $-83^{\circ}$ & \\
\hline 0 & 0 & -1 & 0 & 0 & 0 & & -27.212 & 2.16 & 1.77 & $-83^{\circ}$ & \\
\hline 0 & 0 & -1 & 0 & -1 & 0 & & -27.322 & 13.60 & 11.18 & $-83^{\circ}$ & \\
\hline 0 & 0 & -1 & 0 & -2 & 0 & & -27.432 & 0.70 & 0.57 & $97^{\circ}$ & \\
\hline-1 & 0 & -1 & 2 & -1 & 0 & & -193.56 & 0.63 & 0.56 & $-83^{\circ}$ & \\
\hline 1 & 0 & -1 & 0 & 0 & 0 & & -2190.4 & 1.22 & 1.31 & $-83^{\circ}$ & \\
\hline 1 & 0 & -1 & 0 & -1 & 0 & & -3231.5 & 7.98 & 8.66 & $-83^{\circ}$ & \\
\hline 0 & 0 & 0 & 0 & 0 & 0 & & $\infty$ & 0.74 & 0.83 & $-140^{\circ}$ & \\
\hline-1 & 0 & 1 & 0 & 1 & 0 & & 3231.5 & 9.61 & 11.31 & $97^{\circ}$ & \\
\hline-1 & 0 & 1 & 0 & 0 & 0 & & 2190.4 & 1.62 & 1.95 & $97^{\circ}$ & \\
\hline 0 & 0 & 1 & -1 & 1 & 0 & & 365.24 & 0.89 & 0.91 & $-83^{\circ}$ & \\
\hline-1 & 1 & 1 & 0 & $\mathbf{1}$ & 0 & & 328.17 & 0.53 & 0.14 & $-83^{\circ}$ & \\
\hline 1 & 0 & 1 & -2 & $\mathbf{1}$ & 0 & & 193.56 & 2.83 & 1.46 & $-83^{\circ}$ & \\
\hline 0 & 0 & 1 & 0 & 2 & 0 & & 27.432 & 0.99 & 0.75 & $97^{\circ}$ & \\
\hline 0 & 0 & 1 & 0 & 1 & 0 & & 27.322 & 15.27 & 11.52 & $-83^{\circ}$ & \\
\hline 0 & 0 & 1 & 0 & 0 & 0 & & 27.212 & 2.40 & 1.81 & $-83^{\circ}$ & \\
\hline 1 & 0 & 1 & 0 & 1 & 0 & & 13.719 & 1.15 & 0.87 & $-83^{\circ}$ & \\
\hline \multicolumn{8}{|c|}{ Sum of all amplitudes } & 70.33 & 62.76 & & \\
\hline-1 & 0 & -2 & 0 & -2 & 1 & $Q_{1}$ & 1.11951 & 2.36 & 1.14 & $-120^{\circ}$ & 0.8 \\
\hline 0 & 0 & -2 & 0 & -1 & 1 & & 1.07598 & 2.17 & 1.03 & $-120^{\circ}$ & \\
\hline 0 & 0 & -2 & 0 & -2 & 1 & $O_{1}$ & 1.07581 & 11.56 & 5.47 & $-120^{\circ}$ & 11.3 \\
\hline-1 & 0 & 0 & 0 & $\mathbf{0}$ & 1 & $M_{1}$ & 1.03472 & 0.83 & 0.38 & $60^{\circ}$ & 0.8 \\
\hline 0 & 0 & -2 & 2 & -2 & 1 & $P_{1}$ & 1.00275 & 4.77 & 2.15 & $-120^{\circ}$ & 4.7 \\
\hline 0 & 0 & 0 & 0 & 0 & 1 & $K_{1}$ & 0.99727 & 14.31 & 6.43 & $60^{\circ}$ & 14.6 \\
\hline 0 & 0 & 0 & 0 & -1 & 1 & & 0.99712 & 1.94 & 0.87 & $60^{\circ}$ & \\
\hline 1 & 0 & $\mathbf{0}$ & 0 & 1 & 1 & & 0.96257 & 0.78 & 0.34 & $60^{\circ}$ & \\
\hline \multicolumn{8}{|c|}{ Sum of all amplitudes } & 43.45 & 19.99 & & \\
\hline
\end{tabular}

Eq. (19) can be further refined by expressing $r, \Theta, \Lambda$ as functions of time. Instead, we used the REN2000 nutation tables for a rigid Earth (Souchay et al., 1999) together with the nonrigid-Earth response function given by eq. (17) and assuming $L_{\sigma}=L_{\sigma}^{r}$. Note that this choice of the rigid-Earth nutation theory has no significant influence upon our estimate because according to Bizouard et al. $(2000)$ the agreement between the 3 available theories mentioned in Sec. 1 is at the level of $1 \mu \mathrm{as}$ in the time domain. From the estimated amplitudes of the prograde diurnal polar motion, given in Table 2, we can conclude that: 
- The maximum peak-to-peak variation for the nonrigid Earth is about $40 \mu$ as with the main contributions at frequencies of the principal diurnal tides $K_{1}, O_{1}$ and $P_{1}$.

- Comparison with the ocean-tide contributions (Table 3) shows that prograde diurnal librations are systematically about 25 times smaller and differ in phase by about $+90^{\circ}$.

- Comparison with the prograde diurnal atmospheric tides in polar motion (Table 3) shows similar order of magnitude but different power distribution among diurnal frequencies.

- Our estimate confirms, and even strengthens, the conclusion of Chao et al. (1996) that present measurement and modeling accuracies are inadequate for the diurnal libration in polar motion to close the budget, even for the largest tidal waves.

Table 3. Prograde diurnal and semidiurnal polar motion excited by the oceanic tides (Chao et al., 1996) and by the atmospheric tides (Brzeziński and Petrov, 2000). Periods are in solar days and amplitudes in microarcseconds. Note that the phase is referred to GMST, which differs from the usual convention in representing tidal contributions.

\begin{tabular}{rrrrrrcrrrrr}
\hline \multicolumn{4}{c}{ Fundamental arguments } & & Tidal & Terrestr. & \multicolumn{2}{c}{ Oceanic } & \multicolumn{2}{c}{ Atmospheric } \\
\multicolumn{1}{l}{$l_{m}$} & $l_{s}$ & $F$ & $D$ & $\hat{\Omega}$ & $\phi$ & code & period & ampl. & phase & ampl. & phase \\
\hline-1 & 0 & -2 & 0 & -2 & 1 & $Q_{1}$ & 1.1195 & 27 & $167^{\circ}$ & & \\
0 & 0 & -2 & 0 & -2 & 1 & $O_{1}$ & 1.0758 & 142 & $160^{\circ}$ & & \\
0 & 0 & -2 & 2 & -2 & 1 & $P_{1}$ & 1.0027 & 56 & $153^{\circ}$ & 1.3 & $-133^{\circ}$ \\
0 & -1 & 0 & 0 & 0 & 1 & $S_{1}$ & 1.0000 & & & 7.1 & $47^{\circ}$ \\
0 & 0 & 0 & 0 & 0 & 1 & $K_{1}$ & 0.9973 & 171 & $-27^{\circ}$ & 1.6 & $117^{\circ}$ \\
0 & 1 & 0 & 0 & 0 & 1 & $\psi_{1}$ & 0.9946 & & & 0.7 & $-135^{\circ}$ \\
\hline-1 & 0 & -2 & 0 & -2 & 2 & $N_{2}$ & 0.5274 & 17 & $-45^{\circ}$ & & \\
0 & 0 & -2 & 0 & -2 & 2 & $M_{2}$ & 0.5175 & 75 & $-64^{\circ}$ & & \\
0 & 0 & -2 & 2 & -2 & 2 & $S_{2}$ & 0.5000 & 29 & $-94^{\circ}$ & 2.9 & $-102^{\circ}$ \\
0 & 0 & 0 & 0 & 0 & 2 & $K_{2}$ & 0.4986 & 7 & $-84^{\circ}$ & & \\
\hline
\end{tabular}

\subsection{Low frequency lunisolar polar motion}

Low frequency lunisolar polar motion corresponding to diurnal nutation, is caused by the interaction of the lunisolar tidal potential with the $C_{n, 1}, S_{n, 1}$ $(n \geq 3)$ terms of geopotential, where only the $n=3$ contributions are larger than $0.75 \mu$ as. Detailed expansion (Table 2) shows that the maximum peak-topeak variation for the nonrigid Earth can reach $120 \mu \mathrm{as}$, which is about 3 times larger than prograde diurnal libration and significant in view of the current accuracy of monitoring polar motion. Individual contributions are not exactly at the frequencies of important low frequency tides and are well separated from the much larger atmospheric contributions at seasonal frequencies. The spectrum is approximately symmetrical with respect to zero frequency. Therefore the waves are highly elliptical. It can be seen when writing the largest waves explicitly:

$27.32 \mathrm{~d}: e^{-i 83^{\circ}}[22.7 \mu$ as $\cos (F+\hat{\Omega})+0.3 \mu$ as $\sin (F+\hat{\Omega})]$,

$27.21 \mathrm{~d}: e^{-i 83^{\circ}} 3.6 \mu$ as $\cos F$,

$8.85 \mathrm{y}: \quad e^{-i 83^{\circ}}\left[20.0 \mu\right.$ as $\cos \left(F+\hat{\Omega}-l_{m}\right)+2.6 \mu$ as $\left.\sin \left(F+\hat{\Omega}-l_{m}\right)\right]$. 
Comparison with the ocean-tide contribution by the $M_{m}$ wave done in Table 4 shows that the direct lunisolar effect at a monthly period is only about 4 to 5 times smaller and is of a size similar to the current accuracy of determinations of the amplitudes from the geodetic observations.

Table 4. Comparison of the direct lunisolar effect in polar motion at a monthly period with the ocean-tide contribution and with the observed polar motion with atmospheric contribution (PM/AAM) subtracted, reported by Gross et al. (1998). Amplitudes are in microarcseconds.

\begin{tabular}{lrrrr} 
& \multicolumn{2}{c}{ Retrograde } & \multicolumn{2}{c}{ Prograde } \\
& ampl. & phase & ampl. & phase \\
\hline This work (27.43d) & 0.6 & $97^{\circ}$ & 0.8 & $97^{\circ}$ \\
This work (27.32d) & 11.2 & $-83^{\circ}$ & 11.5 & $-83^{\circ}$ \\
This work (27.21d) & 1.8 & $-83^{\circ}$ & 1.8 & $-83^{\circ}$ \\
\hline Ocean $M_{m}$ tide (27.55d) & & & & \\
Brosche/Seiler/Gross & 55 & $28^{\circ}$ & 53 & $-106^{\circ}$ \\
Dickman's model & 17 & $-7^{\circ}$ & 32 & $-44^{\circ}$ \\
\hline PM/AAM data $M_{m}$ & 59 & $-83^{\circ}$ & 44 & $-85^{\circ}$ \\
& \pm 18 & $\pm 17^{\circ}$ & \pm 20 & $\pm 26^{\circ}$ \\
\hline
\end{tabular}

\section{Conclusions}

Diurnal and sub-diurnal terms of nutation that are caused by the interaction of the lunisolar tidal potential with those geopotential components which express departures from the rotational symmetry, are of geophysical interest because their amplitudes depend on the large-scale features of the core-mantle boundary. We developed an analytical model of the short-period nutation for a nonrigid Earth, based on the rigid-Earth nutation theory REN2000 assuming that the Earth consists of an elastic mantle and a liquid core with no coupling between these media. This model is expressed in the terrestrial frame, that is as polar motion, which is consistent with the treatment of the oceanic and atmospheric effects appearing at similar frequencies. When taking into account the size of various components, we can conclude the following:

- The prograde semidiurnal polar motion corresponding to the terdiurnal nutation is of the order of $0.01 \mu \mathrm{as}$, and therefore completely negligible.

- The prograde diurnal polar motion, sometimes referred to as diurnal libration, corresponding to the semidiurnal nutation has a maximum peak-to-peak size of about $40 \mu$ as. There is a little chance that in the near future this motion can be observationally separated from the much larger ocean-tide influences appearing at the same frequencies.

- The long-period polar motion corresponding to the diurnal nutation, with main constituents at 27.3 days and 8.85 years, is of the maximum peak-to-peak size of about $120 \mu \mathrm{as}$. It is significant in view of the current accuracy of space geodetic observations, and can be separated from the atmospheric and oceanic perturbations. Therefore it should be accounted for in the excitation balance of polar motion. 
Acknowledgments. This research has been supported by the Polish National Committee for Scientific Research (KBN) under grant No. 9 T12E 01315. I am thankful for the IAU travel grant which partly covered the participation costs in the Colloquium.

\section{References}

Bizouard, Ch., Folgueira, M. and Souchay J., 2000, "Comparison of the short period rigid Earth nutation series," in Proc. IAU Colloquium 178, Publications of the Astron. Soc. of the Pacific, Conf. Series, 613-617.

Bretagnon, P., Francou, G., Rocher, P. and Simon, J.-L., 1998, "SMART97: a new solution for the rotation, of the rigid Earth," Astron. Astrophys., 329, 329-338.

Bretagnon, P., 1998, "Diurnal and sub-diurnal nutations for a rigid earth," in Proc. Journées Systèmes de Réf. Spatio-Temporels 1998, Paris Observatory, 164-168.

Brzeziński, A., 1992, "Polar motion excitation by variations of the effective angular momentum function: considerations concerning deconvolution problem." Manuscripta geodaetica, 17, 3-20.

Brzeziński, A. and Capitaine, N., 1993, "The use of the precise observations of the Celestial Ephemeris Pole in the analysis of geophysical excitation of earth rotation," J. Geophys. Res., 98, 6667-675.

Brzeziński, A. and Petrov, S. D., 2000, "High frequency atmospheric excitation of earth rotation," IERS Technical Note No. 28, Paris Observatory, in press.

Chao, B. F., Dong, D. N., Liu, H. S. and Herring, T. A., 1991, "Libration in the earth's rotation," Geophys. Res. Letters, 18, 2007-2010.

Chao, B. F., Ray, R. D., Gipson, M. J., Egbert, G. D. and Ma, C., 1996, "Diurnal/semidiurnal polar motion excited by oceanic tidal angular momentum," J. Geophys. Res., 101, 20,151-20,163.

Dehant, V. and Defraigne, P., 1997, "New transfer functions for nutations of a nonrigid Earth," J. Geophys. Res., B12, 27,659-27,687.

Gross, R. S, Chao, B. F. and Desai, S. D., 1998, "Effect of long-period ocean tides on the earth's polar motion," Prog. Oceanog., 40, 385-397.

Herring, T. A. and Dong, D. N., 1994, "Measurements of diurnal and semidiurnal rotational variations and tidal parameters of Earth," J. Geophys. Res., 99, 18,051-18,071.

Mathews, P. M., Buffett, B. A., Herring, T. A. and Shapiro, I. I., 1991, "Forced nutations of the Earth: Influence of inner core dynamics 2. Numerical results and comparison," J. Geophys. Res., 96, 8243-8257.

McCarthy, D. D. (ed.), 1996, 'IERS Conventions (1996),' IERS Technical Note 21, Observatoire de Paris.

Moritz, H. and Mueller, I. I., 1987, Earth Rotation: Theory and Observation, Ungar Publishing Company, New York. 
Munk, W. H. and MacDonald, G. J. F., 1960, The Rotation of the Earth: A Geophysical Discussion, Cambridge Univ. Press (reprint 1975).

Roosbeek, F., 1999, "Diurnal and subdiurnal terms in RDAN97 series," Cel. Mech. Dyn. Astron., 74, 243-252.

Souchay, J., Loysel, B., Kinoshita, H. and Folgueira, M.: 1999, "Corrections and new developments in rigid earth nutation theory: III. Final tables "REN-2000" including crossed-nutation and spin-orbit coupling effects," Astron. Astrophys. Suppl. Ser., 135, 111-131.

Wahr, J. M., 1981, "The forced nutation of an elliptical, rotating, elastic and oceanless earth," Geophys. J. R. Astr. Soc., 64, 705-727. 